\title{
Human Adaptation Towards a Force Augmenting Device: Experimental Results
}

\author{
S. K. Gadi ${ }^{\text {a, }}$, A. Osorio-Cordero ${ }^{\mathrm{b}}$, Rogelio Lozano ${ }^{\mathrm{b}, \mathrm{c}}$ and R. Garrido ${ }^{\mathrm{d}}$ \\ ${ }^{a}$ FIME, Universidad Autónoma de Coahuila, Torreón, México \\ ${ }^{b}$ UMI-LAFMIA, CINVESTAV-CNRS, Av. IPN 2508., Mexico City, Mexico \\ ${ }^{c}$ Sorbonne Universités, UTC, Heudiasyc UMR 7253, Paris, France \\ ${ }^{d}$ DCA, CINVESTAV, Av. IPN 2508., Mexico City, Mexico
}

\begin{abstract}
This short communication article presents an experiment to test the existence of mental workload (MWL) and adaptability of a human operator $(\mathrm{HO})$ in handling force augmenting devices (FADs). An experiment is conducted where the HOs of three different ages are allowed to handle a FAD with high augmentation factor. Augmentation factor gives the factor by which the HO's force is amplified. It is observed that the HOs were unable to stabilize the FAD for the first time. With the help of these experiments, it is observed that a human adapts itself to achieve a stable HA-FAD interaction. In another perspective, it can be observed that $\mathrm{HO}$ undergoes MWL to handle a FAD with high augmentation factor.
\end{abstract}

Keywords: Force augmenting device, adapting human, ergonomics, human factor, mental workload.

\section{Introduction}

Humanity has employed machines since very long back in time to ease the performance of their tasks. The importance of their usage increases day by day. Before World War II, the machines were designed for a particular purpose, and the human operators had to learn to operate them i.e. human beings had to adapt to the machine. Nowadays, machines are designed to fit the operator characteristics giving birth to the science of ergonomics [1]-[3].

The avoidance of accidents in the human machine interaction (HMI) process is an important concern nowadays. HMI Safety can be improved giving importance to the human factor, e.g. it is observed that the reduction of the stress or mental workload (MWL) in the human operator (HO) contributes significantly to the reduction of accidents [4]-[6].

Humans and machines have different abilities. Human beings have a great variety and a large number of sensors like 3D vision, hearing, touching, etc. These, help humans to perform high precision tasks even in the presence of uncertainties. On the other hand, machines are powerful, can perform repetitive tasks with high precision, without getting tired, can be connected to other machinery to transfer information [7], etc. Exoskeletons and force augmenting devices (FAD) are employed when humans require performing precise tasks that require power that they do not possess. Exoskeletons enhance the abilities of human beings, but the human being is the one who has the

\footnotetext{
* Corresponding author. Tel. \& fax: +521-8717570239

E-mail address: research@ @sgadi.com

Manuscript History:

Received 26 July, 2017, Revised 26 July, 2017, Accepted 21 August, 2017, Published 30 September, 2017
} 
control of the man-machine process. Nowadays there is a growing clinical and industrial interest in the development of machines worn by humans [8]-[13].

A FAD amplifies the human strength and lets the human operator handle heavy loads while still feeling a part of the load [14]. Augmentation factor can be defined as the factor by which human operator's force is amplified. The human-machine interaction has been extensively studied [15]-[24]. In [21], a methodology is presented to calculate the upper limit of augmentation factor for which the human arm (HA)-FAD interaction is stable. However, through numerical simulations, it is observed that changing the parameters internal to the human operator (HO) can stabilize the HA-FAD interaction. This article extends the work presented in [21] by providing experimental results showing that humans indeed adapt themselves to manipulate a FAD.

The next section presents the closed-loop system considered for this experiment. Experimental results are presented in Section 3. Concluding remarks are given in the final section.

\section{Experimental setup}

Figure 1 shows a human operator handling the force augmenting device (FAD). The FAD is made of the following elements.

- A mechanical block of $24 \mathrm{Kg}$ which serves as the load.

- A ball-screw mechanism which converts the rotational movement into linear movement.

- A DC servomotor to power the ball-screw mechanism. The motor's driver is configured to operate in the torque mode. So, the force applied to the moving block is proportional to the control signal.

- Rotary encoder to measure the motor position which allows calculating the position of the mechanical block.

- A force sensor to measure the force applied by the human arm (HA) over the mechanical block. The sensor is attached to the mechanical block.

- A PC with Quanser's data acquisition (DAQ) card is used as a controller for implementing the algorithm in Matlab Simulink. The DAQ lets the computer read the position and force sensor and send the control signal from the computer to the DC motor's driver. 


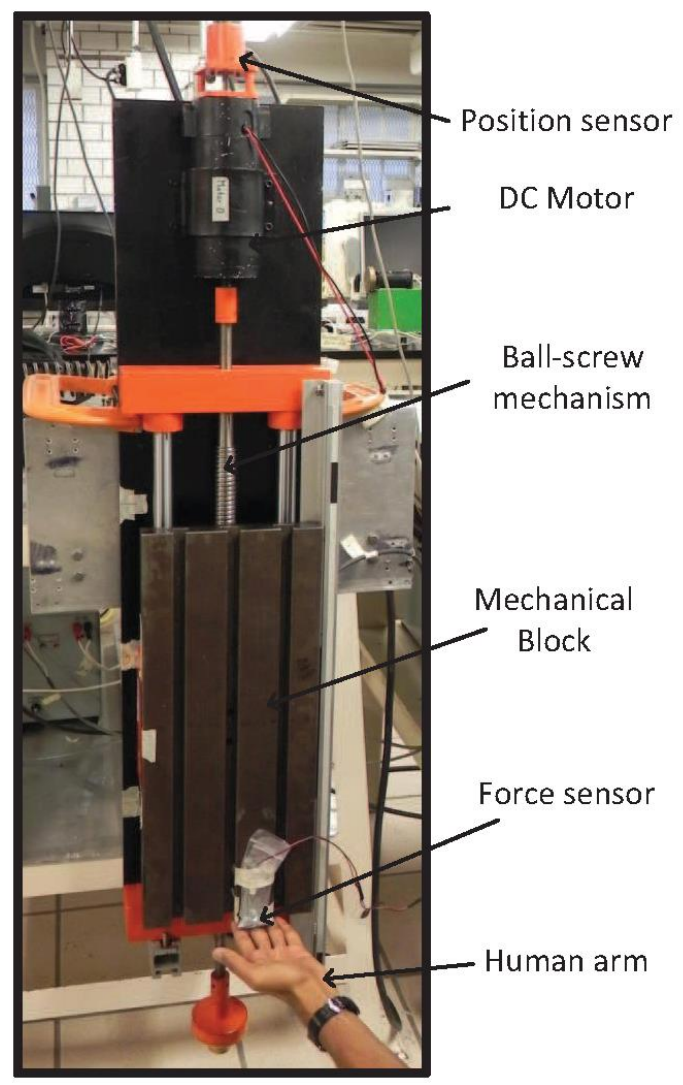

Figure 1. A Human operator is handing the force augmenting device (FAD).

The mathematical model for the HA-FAD is given as [21]:

$$
\begin{aligned}
& F(t)=F_{A}(t)+F_{e}(t)-M g \\
& \frac{y_{e}(s)}{F(s)}=\frac{1}{M s^{2}+K_{f} s} \\
& \tau_{h}(s)=J \ddot{\theta}_{h}(s)+B \dot{\theta}_{h}(s)+K\left(\theta_{h}(s)-\theta_{v}(s)\right), \\
& \theta_{v}(s)=\theta_{v d}(s)+G_{p}\left(\theta_{v d}(s)-\theta_{h}(s) e^{-s d_{1}}\right)+s G_{v}\left(\theta_{v d}(s)-\theta_{h}(s) e^{-s d_{2}}\right), \\
& F_{e}(t)=-F_{h}(t)=\left(y_{h}(t)-y_{e}(t)\right) E \\
& y_{h}(t)=\theta_{h}(t) l_{a} \\
& \tau_{h}(t)=F_{h}(t) l_{a}=\left(y_{e}(t)-y_{h}(t)\right) E l_{a},
\end{aligned}
$$

where $F$ is the total force exerted on the moving block, $F_{e}$ is the force exerted by the human on the moving block, $M$ is the mass of the moving block, $g$ is the acceleration due to gravity, $y_{e}$ is the position of the mechanical block, $s$ is the Laplace transform complex variable, $K_{f}$ is the viscous friction coefficient of $\mathrm{FAD}, \tau_{h}$ is the external torque acting on the human joint, $J$ is the human arm moment of inertia, $\theta_{h}$ is the human arm position, $B$ is the viscous friction in the human arm 
movement, $K$ is the muscle stiffness, $\theta_{v}$ is the output of the spinal cord's reflex action, $\theta_{v d}$ is the virtual desired position, $G_{p}$ and $G_{v}$ are the control parameters of the spinal cord, $d_{1}$ and $d_{2}$ are the delays in the position and velocity reflex feedbacks respectively, $F_{h}$ is the force exerted by the moving block on the human arm, $y_{h}$ is the human arm displacement at the end of the arm, $l_{a}$ is the human arm length, and $E$ is the physical compliance of the human flesh.

The control algorithm is given by

$$
F_{A}(t)=\left(K_{A}-1\right) F_{e}(t)-K_{d} \dot{y}_{e}(t)-K_{p} y_{e}(t)
$$

where $K_{A}>0$ is the augmenting factor that amplifies the force exerted by the human operator, the term $K_{d} \dot{y}_{e}(t)>0$ introduces the required damping into the system, and $K_{p}>0$ the is proportional gain [21]. It is proved that the HA-FAD interaction is stable for all the values of $K_{A}$ provided that $d_{1}=d_{2}=0$ [21]. In the case where delays are not zero, there is an upper limit for the augmenting factor $K_{A}$. A strategy to calculate the upper limit is also presented in [21].

Experiments are conducted on this FAD to verify that the human adapts to stabilize the system. The experimental procedure and its results are discussed in the next section.

\section{Experimental results}

\subsection{Objective and methodology}

An HA-FAD interaction has the following two controllers working in parallel:

1. HO's internal controller: The central nervous system (CNS) and the reflexes of HO act as the HO's internal controller, which actuates the muscles. Equations (3) and (4) presents this controller.

2. Controller external to the HO: A computer is used to actuate the actuators of a FAD as given in (8).

The objective of the experiment is witnessing an HO's internal controller adapting itself to achieve a stable HA-FAD interaction. The following experimental steps are followed in this work:

1. Select the parameters of the controller external to the HO such that the HA-FAD interaction goes unstable when any $\mathrm{HO}$ interacts with the FAD for the first time. An unstable interaction is achieved by selecting very high values of the augmentation factor $K_{A}$.

2. Let the user interact with the FAD sufficient number of times until a stable interaction is achieved.

The experimental setup is shown in Figure 1 and described in the previous section. The experimental setup doesn't physically constrain the user to be in contact with the device all the times, which allows the user to withdraw his arm at any moment. Hence, the experimental setup is safe even under the unstable conditions. The control algorithm (8) is implemented with a very high augmentation factor of $K_{A}=100 \times 10^{3}$. 
HOs of three age groups are selected to verify that these results are consistent across all the age groups. HOs are instructed to release the hand after few seconds to avoid the fatigue affecting interaction performance. HOs were left free to choose the time for which they could interact the FAD.

\subsection{Results and discussion}

Figure 2, 3 and 4 show the experimental results conducted on three healthy individuals of age groups 10, 20 and 30. Individuals are instructed to lift the mechanical block and hold steadily at any position for as long as they could interact with FAD comfortably without feeling fatigue. Figure 2(a), 3(a) and 4(a) shows the HA-FAD interaction where the users handle the FAD for the first time. It is observed that they were unable to hold the mechanical block at a constant position, which reflects that the HA-FAD interaction is unstable.

The individuals are asked to repeat the process until they could achieve a stable interaction with the FAD. The third time HA-FAD interactions are recorded and displayed in Figure 2(b), 3(b) and 4(b). These graphs show that the interactions are stable from the third time onwards, which suggest that the HO's internal controller adapted itself to stabilize the interaction. $G_{p}$ and $G_{v}$ are the only parameters which are capable of adapting. Hence, when an interaction goes unstable, CNS updates the parameters $G_{p}$ and $G_{v}$, which is imposing MWL on the HO. Therefore, it implies that higher values of augmentation factor or possible unstable conditions cause MWL on the HOs.

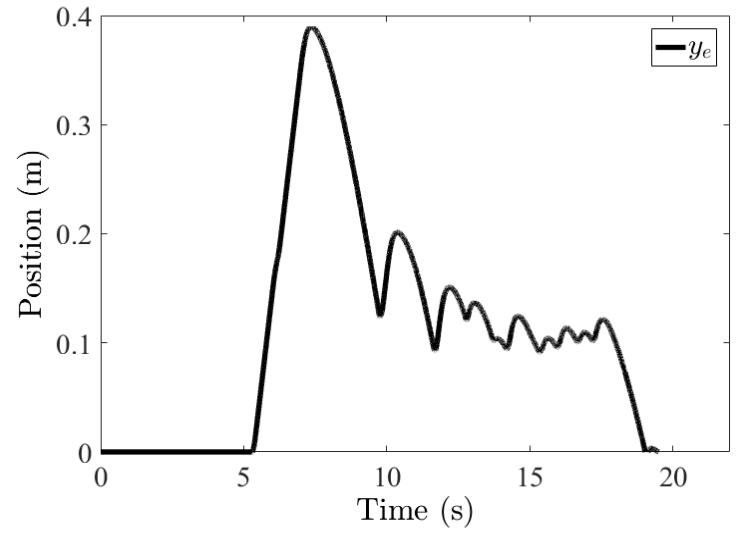

(a) Handling the FAD for the first time

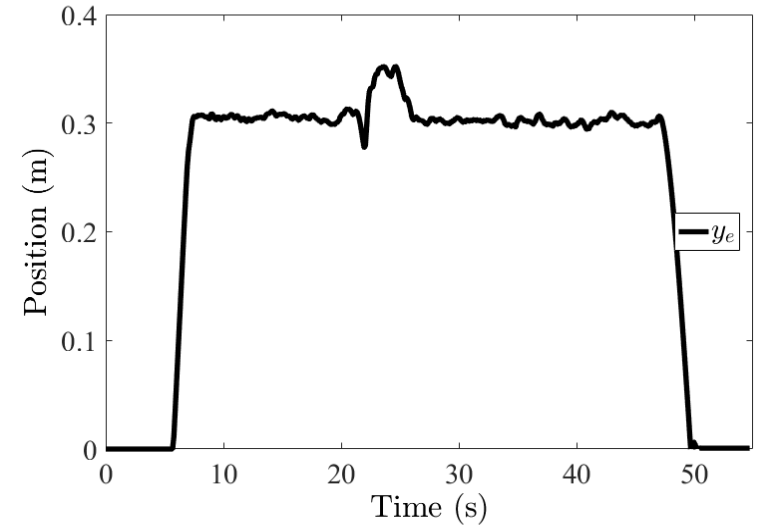

(b) Handling the FAD for the third time

Figure 2. A 10-year-old individual handing the FAD with $K_{A}=100 \times 10^{3}$ 


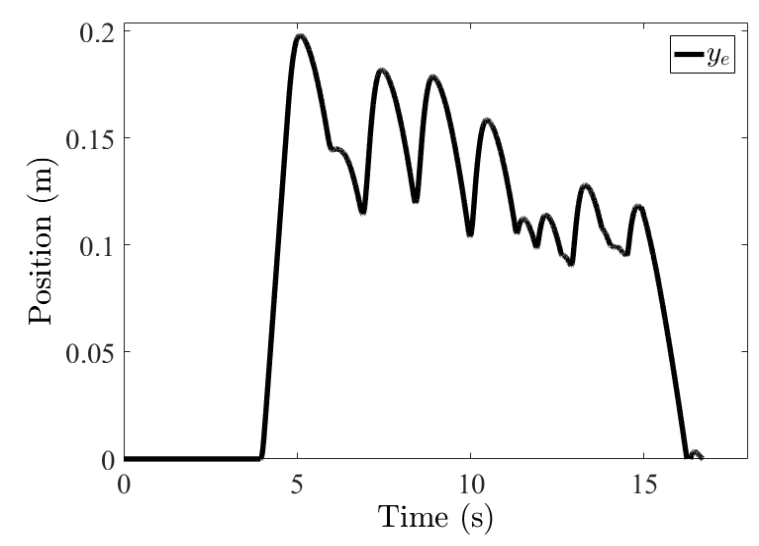

(a) Handling the FAD for the first time

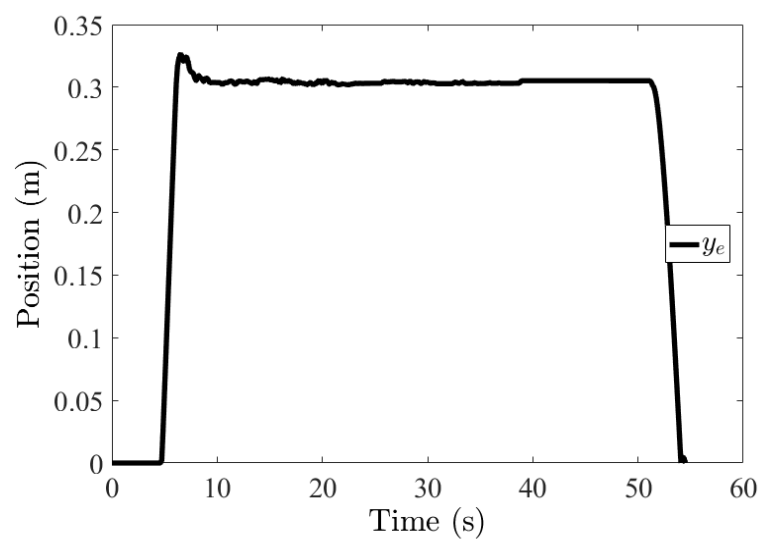

(b) Handling the FAD for the third time

Figure 3. A 20-year-old individual handing the FAD with $K_{A}=100 \times 10^{3}$

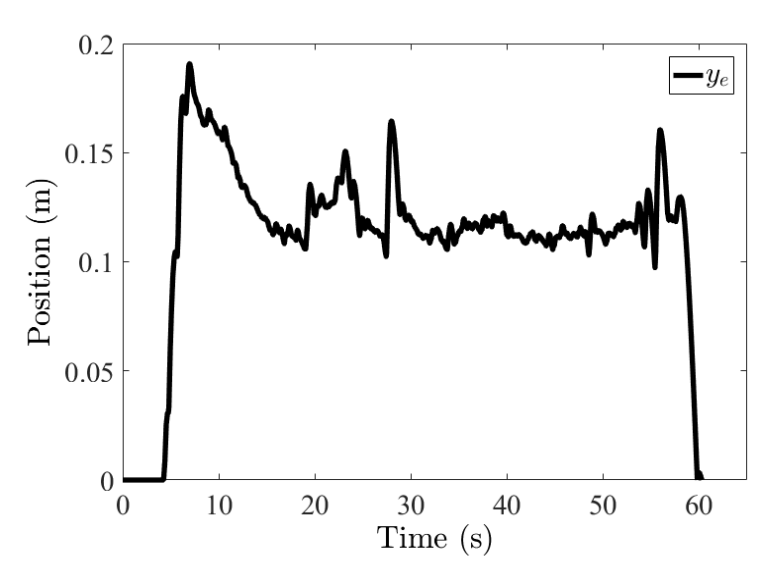

(a) Handling the FAD for the first time

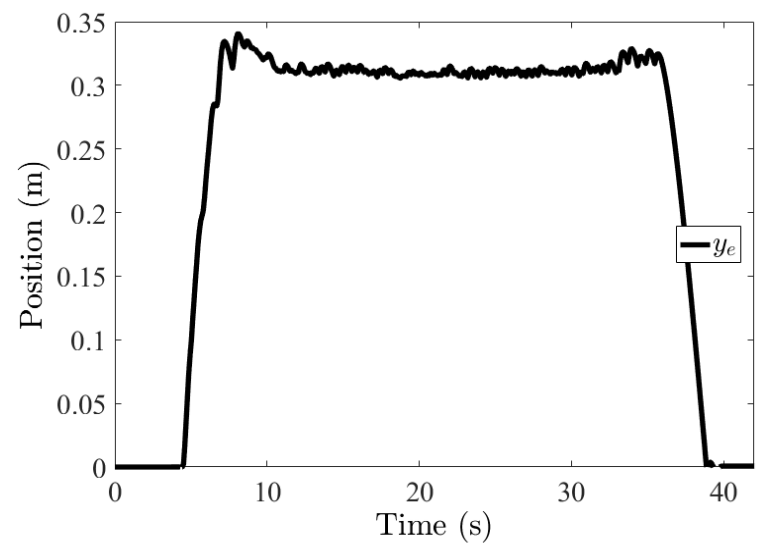

(b) Handling the FAD for the third time

Figure 4. A 30 -year-old individual handing the FAD with $K_{A}=100 \times 10^{3}$

\section{Conclusions}

This work extends [21] to investigate the adapting capability of the human operator (HO). In the light of the experimental results presented in the previous section, it can be concluded that the human operator adapts to stabilize the interaction. Since the parameters $d_{1}$ and $d_{2}$ cannot be changed, the parameters $G_{p}$ and $G_{v}$ associated with the human operator's reflex action are capable of adapting. The simulation results presented in [21] also suggest that by selecting the parameters $G_{p}$ and $G_{v}$ an unstable interaction can be converted to a stable one.

It is observed that for higher values augmentation factor $K_{A}$, $\mathrm{HO}$ is unable to attain a stable HA-FAD interaction. Hence, it can be considered that HO undergoes a mental workload (MWL) to stabilize the interaction. These results confirm the importance of considering human factor into the FAD's design to reduce the MWL on the human operator. It is seen that HO was able to maintain a stable interaction with the FAD after three attempts, i.e. HO's undergo lesser MWL in their subsequent attempts. So, the authors recommend in using new test subjects for the testing of a FAD with high augmentation factor. 


\section{Acknowledgements}

The authors would like to thank Gerardo Castro, and Jesús Meza, for their help in implementing the electrical setup and Roberto Lagunes for his help in building the mechanical setup.

\section{References}

[1] Charlton, S. G., and O'Brien, T. G. (Eds.). (2001). Handbook of Human Factors Testing and Evaluation. CRC Press.

[2] Pheasant, S., and Haslegrave, C. M. (2016). Bodyspace: Anthropometry, Ergonomics and the Design of Work. CRC Press.

[3] Young, M. S., Brookhuis, K. A., Wickens, C. D., and Hancock, P. A. (2015). State of Science: Mental Workload in Ergonomics. Ergonomics, 58(1), 1-17.

[4] Flaspöler, E., Hauke, A., Pappachan, P., Reinert, D., Bleyer, T., Henke, N., and Beeck, R. O. D. (2009). The Human-Machine Interface as an Emerging Risk. EU-OSHA (European Agency for Safety and Health at Work). Luxemburgo.

[5] Brun, E. (2007). Expert Forecast on Emerging Biological Risks Related to Occupational Safety and Health. Office for Official Publications of The European Communities.

[6] Chen, J., Song, X., amd Lin, Z. (2016). Revealing the "Invisible Gorilla" in Construction: Estimating Construction Safety through Mental Workload Assessment. Automation in Construction, 63, 173-183.

[7] Colgate, J. E., Peshkin, M., and Klostermeyer, S. H. (2003). Intelligent Assist Devices in Industrial Applications: A Review. In Intelligent Robots and Systems, 2003.(IROS 2003). Proceedings. 2003 IEEE/RSJ International Conference, 3, 2516-2521.

[8] Guizzo, E., and Goldstein, H. (2005). The Rise of the Body Bots [Robotic Exoskeletons]. IEEE spectrum, 42(10), 50-56.

[9] Kim, W. S., Lee, S. H., Lee, H. D., Yu, S. N., Han, J. S., \& Han, C. S. (2009). Development of the Heavy Load Transferring Task Oriented Exoskeleton Adapted by Lower Extremity Using Qausi-Active Joints. In ICCAS-SICE, 2009 1353-1358.

[10] Gopura, R. A. R. C., Bandara, D. S. V., Kiguchi, K., and Mann, G. K. (2016). Developments in Hardware Systems of Active Upper-Limb Exoskeleton Robots: A Review. Robotics and Autonomous Systems, 75, 203-220.

[11] Miller, L. E., Zimmermann, A. K., and Herbert, W. G. (2016). Clinical Effectiveness and Safety of Powered Exoskeleton-Assisted Walking in Patients with Spinal Cord Injury: Systematic Review with Meta-Analysis. Medical Devices (Auckland, NZ), 9, 455-456.

[12] Yan, T., Cempini, M., Oddo, C. M., and Vitiello, N. (2015). Review of Assistive Strategies in Powered Lower-Limb Orthoses and Exoskeletons. Robotics and Autonomous Systems, 64, 120-136.

[13] Sheridan, T. B. (2016). Human-Robot Interaction: Status and Challenges. Human factors, 58(4), 525532.

[14] Snyder T. J. and H. Kazerooni, “A Novel Material Handling System,” in IEEE International Conference on Robotics and Automation, 1996. Proceedings., 1996, 2, 1147-1152.

[15] Operation, S. M. H. P. (1969). Hardiman 1 Prototype Project. Tech. Rep.

[16] Kazerooni, H. (1990). Human-Robot Interaction via the Transfer of Power and Information Signals. IEEE Transactions on Systems, Man, and Cybernetics, 20(2), 450-463.

[17] Lee, S., and Sankai, Y. (2002). Power Assist Control for Leg with Hal-3 Based on Virtual Torque and Impedance Adjustment. In Systems, Man and Cybernetics, 2002 IEEE International Conference, 4, 6.

[18] Yamamoto, K., Hyodo, K., Ishii, M., and Matsuo, T. (2002). Development of Power Assisting Suit for Assisting Nurse Labor. JSME International Journal Series C Mechanical Systems, Machine Elements and Manufacturing, 45(3), 703-711.

[19] Kazerooni, H., and Steger, R. (2006). The Berkeley Lower Extremity Exoskeleton. Journal of dynamic systems, measurement, and control, 128(1), 14-25.

[20] Kong, K., and Tomizuka, M. (2009). Control of Exoskeletons Inspired by Fictitious Gain in Human Model. IEEE/ASME Transactions on Mechatronics, 14(6), 689-698.

[21] Gadi, S. K., Osorio-Cordero, A., Lozano-Leal, R., and Garrido, R. A. (2017). Stability Analysis of a Human Arm Interacting with a Force Augmenting Device. Journal of Intelligent \& Robotic Systems, 86(2), 215-224. 
[22] Gradetsky, V. G., Ermolov, I. L., Knyazkov, M. M., Semyonov, E. A., and Sukhanov, A. N. (2014). Experimental Investigation of Human Exoskeleton Model. In Advances on Theory and Practice of Robots and Manipulators, 275-281.

[23] Manns, P., Sreenivasa, M., Millard, M., and Mombaur, K. (2017). Motion Optimization and Parameter Identification for a Human and Lower Back Exoskeleton Model. IEEE Robotics and Automation Letters, 2(3), 1564-1570.

[24] Simkins, M. (2016). A Physiologically Based Hypothesis for Learning Proprioception and In Approximating Inverse Kinematics. Physiological Reports, 4(10), e12774. 